\title{
NECESSIDADES DOS CUIDADORES INFORMAIS DA PESSOA COM DEMÊNCIA EM CONTEXTO DE INSTITUCIONALIZAÇÃO DE LONGO PRAZO: REVISÃO SCOPING
}

\author{
Júlio Belo Fernandes \\ Escola Superior de Saúde Egas Moniz \\ Centro de Investigação Interdisciplinar Egas Moniz \\ juliobelo@sapo.pt \\ Diana Alves Vareta \\ Instituição: Instituto Politécnico de Setúbal \\ Escola Superior de Saúde \\ Florencio Vicente Castro \\ Profesor Doctor Catedrático en la Facultad de Educación \\ Universidad de Extremadura
}

Recepción Artículo: 10 febrero 2020

Admisión Evaluación: 4 marzo 2020

Informe Evaluador 1: 13 marzo

Informe Evaluador 2: 17 marzo 2020

Aprobación Publicación: 20 abril 2020

\section{RESUMO}

Enquadramento: Assumir a decisão de institucionalizar a pessoa com demência é uma situação que desencadeia stress nos cuidadores informais, verificando-se relatos de sentimentos de perda de controlo, solidão, isolamento, culpabilização e vergonha por não conseguirem manter o papel de cuidadores.

É fundamental conhecer quais são as necessidades sentidas pelos cuidadores informais da pessoa com demência em contexto de institucionalização de longo prazo, de modo a poder ser realizada uma intervenção centrada na pessoa, englobando o seu familiar como parte do cuidar.

Objetivo: Identificar as necessidades dos cuidadores informais da pessoa com demência em contexto de institucionalização de longo prazo.

Método: Revisão Scoping desenvolvida sob as orientações do Joanna Briggs Institute, com recurso a pesquisa no motor de busca EBSCO, nas bases de dados MEDLINE e CINAHL, e na base de dados ScienceDirect, com o friso temporal de 2009 a 2018.

Resultados: Foram selecionados seis artigos que envolveram cuidadores de pessoas com demência em contexto de institucionalização de longo prazo. Verificou-se que existem necessidades distintas nas fases pré e pós-institucionalização. A pré-institucionalização relacionada com o processo de tomada de decisão de institucionalizar a pessoa, onde se incluem as necessidades de conhecer previamente a instituição e a existência de uma rede de apoio que suporte a decisão. A pós-institucionalização, diz respeito a todas as necessidades que surgem desde 0 momento de admissão da pessoa na instituição de cuidados a longo prazo, nomeadamente 0 acolhimento, 0 apoio durante as visitas, 0 estabelecimento de uma parceria com os cuidadores formais, a monitorização da qualidade dos cuidados e as questões financeiras. 


\title{
NECESSIDADES DOS CUIDADORES INFORMAIS DA PESSOA COM DEMÊNCIA EM CONTEXTO DE INSTITUCIONALIZAÇÃOO DE LONGO PRAZO: REVISÃO SCOPING
}

Conclusão: A institucionalização da pessoa com demência desencadeia nos cuidadores informais novos focos de necessidades que devem ser alvo de intervenção por parte dos profissionais de saúde, de modo a facilitar o processo de transição subjacente.

Palavras-chave: cuidador; família; determinação de necessidades de cuidados de saúde; demência; institucionalização; cuidados a longo prazo

\begin{abstract}
Needs of caregivers of people with dementia in the context of long-term institutionalization: Scoping review. Background: Taking the decision to institutionalize the person with dementia is a situation that triggers stress in caregivers, with reports of feelings of loss of control, Ioneliness, isolation, blame and shame for not being able to maintain the role of caregivers. It is essential to know what are the needs felt by the caregivers of the person with dementia in the context of long-term institutionalization, so that a person-centered intervention can be carried out, encompassing the family members as part of the care.

Objective: To identify the needs of caregivers of people with dementia in the context of long-term institutionalization.

Methodology: Scoping review developed under the guidelines of the Joanna Briggs Institute, using the databases MEDLINE, CINAHL and ScienceDirect, with the time frame from 2009 to 2018.

Results: Six articles were selected that involved caregivers of people with dementia in the context of longterm institutionalization. It was found that there are different needs in the pre and post-institutionalization phases. Pre-institutionalization relates to the decision-making process of institutionalizing the person, which includes the needs to know the institution in advance and the existence of a network that supports the decision. Post-institutionalization refers to the needs that arise from the moment the person is admitted to the institution of long-term care, namely the reception, support during visits, the establishment of a partnership with health professionals, monitoring quality of care and financial issues.
\end{abstract}

Conclusion: The institutionalization of the person with dementia triggers in the caregivers new focuses of needs that should be the target of intervention by health professionals, in order to facilitate the underlying transition process.

Keywords: caregivers; family; needs assessment; dementia; institutionalization; Iong-term care

\section{INTRODUÇÃo}

Denomina-se por demência o conjunto de condições que se caracterizam pela deterioração da capacidade cognitiva e funcional de forma progressiva (Thompson, \& Roger, 2014).

A demência tem vindo a constituir um problema de saúde crescente e com grande impacto social e económico a nível mundial. Num estudo realizado em 2015, com foco na demência a nível global, foi estimado existir 46,8 milhões de pessoas com demência em todo o mundo, com previsão de em 2050 este número ser três vezes superior (Prince et al., 2015). Em Portugal, Santana, Farinha, Freitas, Rodrigues e Carvalho (2015) relatam existir 160287 pessoas com mais de 60 anos de idade com o diagnóstico de demência. Segundo os dados do relatório da Organização para Cooperação e Desenvolvimento Económico (2017), dos países que englobam esta organização Portugal é o $4^{0}$ país com maior prevalência de demência, com 19.9 casos por mil habitantes, comparativamente com a média de 14.8 casos por mil habitantes.

0 declínio da autonomia e da capacidade funcional da pessoa com demência condicionam o equilíbrio familiar. Para o cuidador informal assumir esta função implica uma mudança no seu modo de vida, que se traduz numa realidade muito mais complexa que 0 apoio prestado a pessoas dependentes na realização das atividades de vida diárias (Hoffman, \& Rodrigues, 2010). Esta transição constitui um evento significativo com repercussões a diferentes níveis, quer pelas dimensões física e psicológica, como pela duração prolongada no tempo em que decorre. Está relacionada com a possibilidade de diminuiç̧ão das interações sociais, de mudanças nos relacionamentos e com a necessidade de reorganização da vida pessoal (Pereira, 2011). 
Do impacto que a demência pode causar nos cuidadores informais, um estudo realizado por Pinquart e Sörensen (2003) demonstrou que estes apresentam níveis mais elevados de stress, de depressão e problemas físicos, quando comparados com cuidadores informais de pessoas sem demência. Também Joling et al. (2015) realizaram um estudo longitudinal numa população de cuidadores informais de pessoas com demência e identificaram incidências de $37 \%$ de depressão e $55 \%$ de perturbações da ansiedade.

A decisão de institucionalização da pessoa com demência surge muitas vezes em resposta ao elevado volume de cuidados, no entanto está associada ao aumento dos sentimentos de sobrecarga e depressão para alguns cuidadores, como foi identificado no estudo realizado por Gaugler, Mittelman, Hepburn e Newcomer (2009). 0 processo de institucionalização constitui uma transição para o cuidador e para a pessoa, sendo caracterizado por diferentes fases e mudanças, traduzindo-se num período conturbado para ambos (Meleis, 2010). Esta transição surge frequentemente em resposta a mudanças no estado de saúde, em consequência do incremento de necessidades de cuidados, e quando estas excedem os recursos e capacidades no seio familiar (Hainstock, Cloutier, \& Penning, 2017).

Para os cuidadores, a institucionalização não significa o término do cuidado à pessoa com demência, estes revelam preocupações emocionais como insegurança, sentimento de culpa e tristeza com a decisão após a admissão (Afram, Verbeek, Bleijlevens, \& Hamers, 2015).

A transição para uma instituição desencadeia mudanças significativas na vida da pessoa com demência, é uma experiência marcante que representa uma perda nas ligações a vizinhos, família e amigos e ao ambiente confortador da sua casa, o que irá originar alterações comportamentais e dificuldades na aceitação do novo contexto (Sury, Burns, \& Brodaty, 2013).

Dada a natureza transacional desta problemática e ao reconhecer que o estabelecimento de uma parceria com o cuidador informal é essecial para a prestação de cuidados de qualidade, é fulcral que os profissionais de saúde tenham presentes as necessidades dos cuidadores informais da pessoa com demência em contexto de institucionalização de longo prazo. Assim, procedeu-se à realização desta revisão scoping, que tem como objetivo identificar as necessidades dos cuidadores informais da pessoa com demência em contexto de institucionalização de longo prazo.

\section{METODOLOGIA}

De forma a detalhar o processo metodológico a percorrer, foi desenvolvido um protocolo de pesquisa, considerando as orientações do Joanna Briggs Institute (2015) para a elaboração de revisões scoping. A questão de investigação que norteou a pesquisa, foi desenvolvida no formato participants, concept e context (PCC): Quais são as necessidades $(C)$ dos cuidadores informais da pessoa com demência $(P)$ em contexto de institucionalização de longo prazo (C)?

As etapas de pesquisa, seleção e extração dos dados foram efetuadas por dois investigadores de forma independente.

\section{Estratégia de pesquisa}

De modo a avaliar a pertinência da realização desta revisão scoping foi realizada uma pesquisa exploratória, com recurso às bases de dados: JBI Evidence-based Database; EBSCO host (CINAHL Plus with Full Text, MEDLINE with Full Text); ScienceDirect; Nursing Reference Center; Biblioteca do conhecimento online; Google Académico.

Tendo verificado a não existência de uma revisão que responda à questão de investigação, procedeu-se à sua elaboração recorrendo à plataforma EBSCOhost, bases de dados CINAHL Plus with Full Texte MEDLINE with Full Text, e ScienceDirect, utilizando os limitadores: disponibilidade de artigos em texto integral; publicados entre janeiro de 2009 e dezembro 2018; redigidos em português, inglês ou espanhol.

A estratégia de pesquisa foi: $(P)$ [carers OR caregivers OR family member OR dementia] AND $(C)$ [needs assessment] AND (C) [long-term care OR nursing home]. 


\section{NECESSIDADES DOS CUIDADORES INFORMAIS DA PESSOA COM DEMÊNCIA EM CONTEXTO DE INSTITUCIONALIZAÇÃO DE LONGO PRAZO: REVISÃO SCOPING}

\section{Seleção dos artigos}

0 processo de seleção dos artigos foi desenvolvido em três fases, tendo em consideração a questão de investigação e os limitadores definidos. A primeira fase incluiu a análise da pertinência dos documentos identificados com base no título, seguindo-se a análise do resumo e, por último, efetuou-se a leitura integral dos documentos selecionados.

Foram obtidos 633 artigos, dos quais se eliminaram 26 por repetição e 573 após a leitura do título e resumo, tendo resultado 34 artigos para leitura integral. Destes, 28 foram excluídos por não responderem à questão de investigação elaborada para esta revisão. Após consenso entre os revisores foi determinada a seleção de 6 artigos.

Relativamente aos documentos não indexados/ publicados, foram utilizados os mesmos limitadores, no entanto não foram identificados novos documentos.

Para a apresentação destas etapas, foi desenvolvido o seguinte Prisma Flow (Figura 1).

Figura 1 - Diagrama Prisma Flow

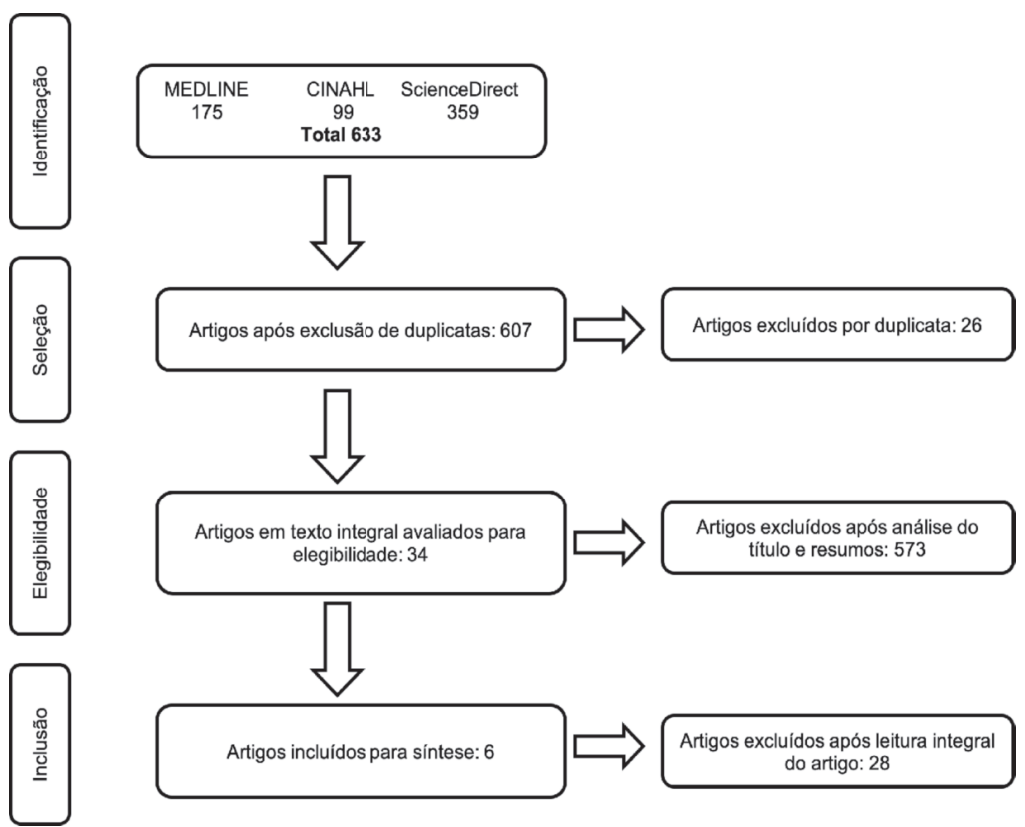

\section{Extração de dados}

0 processo de extração dos dados foi efetuado tendo por base um instrumento de colheita de dados, sendo essas informações, posteriormente transferidas para um quadro de extração de dados (Quadro 1). A síntese dos dados recolhidos nos artigos foi organizada de acordo com o autor e ano de publicação, título do artigo, objetivo do estudo e resultados relacionados com as necessidades de cuidados. 
Quadro 1 - Extração de dados

\begin{tabular}{|c|c|c|}
\hline & & os \\
\hline $\begin{array}{l}\text { Johansson, Ruzin, } \\
\text { Graneheim, \& Lindgren, } \\
2014 \\
\text { Remaining connected } \\
\text { despite separation - } \\
\text { former family caregivers' } \\
\text { experiences of aspects that } \\
\text { facilitate and hinder the } \\
\text { process of relinquishing } \\
\text { the care of a person with } \\
\text { dementia to a nursing } \\
\text { home }\end{array}$ & $\begin{array}{l}\text { Identificar as experiências } \\
\text { dos cuidadores informais } \\
\text { que facilitam ou dificultam } \\
\text { o processo de transferência } \\
\text { do cuidado à pessoa com } \\
\text { demência para uma } \\
\text { unidade de cuidados de } \\
\text { longo prazo. }\end{array}$ & $\begin{array}{l}\text { - Rede de apoio que suporte a decisão de } \\
\text { institucionalizar o familiar; } \\
\text { - Conhecimento prévio da instituição; } \\
\text { - Acolhimento com um ambiente agradável } \\
\text { e profissional; } \\
\text { - Estabelecer uma relação informal e de } \\
\text { proximidade com os profissionais; } \\
\text { - Ser reconhecido como porta-voz do } \\
\text { familiar; } \\
\text { - Ver reconhecido o seu valor/ experiência; } \\
\text { - Monitorizar: } \\
\text { o qualidade dos cuidados; } \\
\text { o formação dos profissionais. }\end{array}$ \\
\hline $\begin{array}{l}\text { Graneheim, Johansson, \& } \\
\text { Lindgren, } 2014 \\
\text { Family caregivers' } \\
\text { experiences of } \\
\text { relinquishing the care of a } \\
\text { person with dementia to a } \\
\text { nursing home: insights } \\
\text { from a meta-ethnographic } \\
\text { study }\end{array}$ & $\begin{array}{l}\text { Descrever as experiências } \\
\text { dos cuidadores informais } \\
\text { da pessoa com demência } \\
\text { após a institucionalização } \\
\text { do seu familiar. }\end{array}$ & $\begin{array}{l}\text { - Rede de apoio que suporte a decisão de } \\
\text { institucionalizar o familiar; } \\
\text { - Conhecimento prévio da instituição; } \\
\text { - Gestão de tempo/ conciliar a vida familiar, } \\
\text { profissional e visitas regulares ao familiar; } \\
\text { - Gestão financeira; } \\
\text { - Ser reconhecido como porta-voz do } \\
\text { familiar; } \\
\text { - Monitorizar: } \\
\text { o qualidade dos cuidados; } \\
\text { o cuidado personalizado. }\end{array}$ \\
\hline 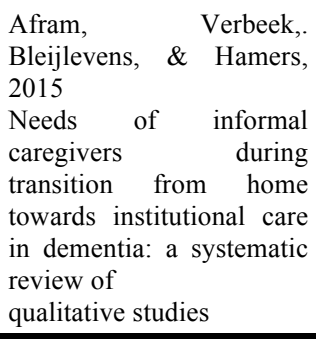 & $\begin{array}{l}\text { Fornecer informações } \\
\text { sobre os problemas e as } \\
\text { necessidades dos } \\
\text { cuidadores informais de } \\
\text { pessoas com demência } \\
\text { durante a transição de } \\
\text { cuidados domiciliários } \\
\text { para cuidados } \\
\text { institucionais de longo } \\
\text { prazo. }\end{array}$ & $\begin{array}{l}\text { - Rede de apoio que suporte a decisão de } \\
\text { institucionalizar o familiar; } \\
\text { - Partilha de informação; } \\
\text { - Influenciar o cuidado prestado ao familiar; } \\
\text { - Ambiente de proximidade com os } \\
\text { profissionais; } \\
\text { - Gestão financeira; } \\
\text { - Monitorizar: } \\
\text { o qualidade dos cuidados } \\
\text { orácio clientes/ profissionais. }\end{array}$ \\
\hline $\begin{array}{l}\text { Law, Patterson, \& Muers, } \\
2016 \\
\text { Staff Factors Contributing } \\
\text { to Family Satisfaction } \\
\text { with Long-Term Dementia } \\
\text { Care: A Systematic } \\
\text { Review of the Literature }\end{array}$ & $\begin{array}{l}\text { Avaliar as evidências } \\
\text { empíricas sobre os fatores } \\
\text { relacionados com a equipa } \\
\text { prestadora de cuidados que } \\
\text { contribuem para a } \\
\text { satisfação das famílias de } \\
\text { pessoas com demência em } \\
\text { contexto de } \\
\text { institucionalização. }\end{array}$ & $\begin{array}{l}\text { - Apoio dos profissionais durante as visitas; } \\
\text { - Acolhimento com um ambiente agradável } \\
\text { e profissional; } \\
\text { - Estabelecer uma relação informal e de } \\
\text { proximidade com os profissionais; } \\
\text { - Influenciar o cuidado prestado ao familiar; } \\
\text { - Partilha de informação; } \\
\text { - Ver reconhecido o seu valor/ experiência; } \\
\text { - Monitorizar: } \\
\text { o qualidade dos cuidados } \\
\text { orácio clientes/ profissionais; } \\
\text { o formação dos profissionais; } \\
\text { o rotatividade da equipa; } \\
\text { o cuidado personalizado. }\end{array}$ \\
\hline
\end{tabular}




\begin{tabular}{|c|c|c|}
\hline $\begin{array}{l}\text { Cronfalk, Ternestedt, \& } \\
\text { Norberg, } 2017 \\
\text { Being a close family } \\
\text { member of a person with } \\
\text { dementia living in a } \\
\text { nursing home }\end{array}$ & $\begin{array}{l}\text { Clarificar a experiência } \\
\text { vivida dos familiares de } \\
\text { pessoas com demência, } \\
\text { antes e depois da admissão } \\
\text { do seu familiar numa } \\
\text { unidade de cuidados } \\
\text { institucionais de longo } \\
\text { prazo. }\end{array}$ & $\begin{array}{l}\text { - Rede de apoio que suporte a decisão de } \\
\text { institucionalizar o familiar; } \\
\text { - Gestão de tempo/ conciliar a vida familiar, } \\
\text { profissional e visitas regulares ao familiar; } \\
\text { - Acolhimento com um ambiente agradável } \\
\text { e profissional; } \\
\text { - Estabelecer uma relação informal e de } \\
\text { proximidade com os profissionais; } \\
\text { - Gestão financeira; } \\
\text { - Monitorizar: } \\
\text { o qualidade dos cuidados; } \\
\text { o rácio clientes/ profissionais; } \\
\text { orotatividade da equipa. }\end{array}$ \\
\hline $\begin{array}{l}\text { Müller, Lautenschläger, } \\
\text { Meyer, \& Stephan, } 2017 \\
\text { Interventions to support } \\
\text { people with dementia and } \\
\text { their caregivers during the } \\
\text { transition from home care } \\
\text { to nursing home care: A } \\
\text { systematic review }\end{array}$ & $\begin{array}{l}\text { Identificar as intervenções } \\
\text { de suporte às necessidades } \\
\text { das pessoas com demência } \\
\text { e dos seus cuidadores na } \\
\text { transição do cuidado em } \\
\text { contexto domiciliário para } \\
\text { o institucional; r dessas } \\
\text { Sintetizar as evidências da } \\
\text { eficácia as } \\
\text { intervenções; se arificadas } \\
\text { Examinar identificanente } \\
\text { intervenções idematicamente } \\
\text { foram sisteras. } \\
\text { desenvolvidas. }\end{array}$ & $\begin{array}{l}\text { - Reconhecer que os cuidadores informais } \\
\text { têm necessidades específicas; } \\
\text { - Rede de apoio que suporte a decisão de } \\
\text { institucionalizar o familiar; } \\
\text { - Possuir informações sobre: } \\
\text { o alternativas de cuidado disponíveis; } \\
\text { o opções financeiras; } \\
\text { - necessidades de cuidados potenciais; } \\
\text { - Sentir satisfação com a instituição } \\
\text { escolhida; } \\
\text { - Apoio dos profissionais durante as visitas; } \\
\text { - Estabelecer uma relação de proximidade } \\
\text { com os profissionais. }\end{array}$ \\
\hline
\end{tabular}

\section{RESULTADOS E DISCUSSÃO}

Dos seis artigos selecionados, dois são estudos exploratórios descritivos (Cronfalk, Ternestedt, \& Norberg, 2017; Johansson, Ruzin, Graneheim, \& Lindgren, 2014) e quatro são revisões sistemáticas da literatura (Afram et al., 2015; Graneheim, Johansson, \& Lindgren, 2014; Law, Patterson e Muers, 2016; Müller, Lautenschläger, Meyer, \& Stephan, 2017). Estas investigações são provenientes da Suécia, Holanda, Alemanha e Reino Unido.

Dos estudos exploratórios descritivos, o primeiro foi efetuado por Cronfalk et al. (2017) e teve como objetivo clarificar a experiência vivida dos familiares de pessoas com demência, antes e depois da admissão do seu familiar numa unidade de cuidados institucionais de longo prazo. 0 segundo estudo, desenvolvido por Johansson et al. (2014), pretendeu identificar as experiências dos cuidadores informais que facilitam ou dificultam o processo de transferência do cuidado à pessoa com demência para uma unidade de cuidados de longo prazo.

Das quatro revisões sistemáticas da literatura, Graneheim et al. (2014) teve como objetivo descrever a experiência dos cuidadores informais no processo de institucionalização da pessoa com demência. Afram et al. (2015) pretenderam identificar o conhecimento acerca dos problemas e das necessidades dos cuidadores informais de pessoas com demência durante a transição de cuidados domiciliares para cuidados institucionais de longo prazo. Law et al. (2016) desenvolveram uma investigação com o objetivo de identificar quais são os fatores relacionados com a equipa prestadora de cuidados que contribuem para a satisfação do familiar da pessoa com demência em contexto de institucionalização. Por último, Müller et al. (2017) pretenderam identificar quais são as intervenções de suporte às necessidades dos cuidadores de pessoas com demência na transição de cuidado em contexto domiciliário para o institucional.

Na análise realizada, verificou-se que os cuidadores informais não descrevem o assumir a institucionalização da pessoa com demência como um sentimento de alívio. A redução do encargo fisico e emocional da prestação 
direta de cuidados ao cuidador informal poderia diminuir as suas necessidades pessoais e levar à eliminação da sobrecarga, mas não foi o descrito nos artigos analisados. Verificou-se uma alteração no foco destabilizador, destacando-se o momento que antecede a institucionalização como o de maior carga emocional.

Com 0 intuito de minimizar 0 impacto negativo associado à institucionalização Müller et al. (2017) ressalvam a importância de ir ao encontro das necessidades específicas dos cuidadores. Conhecer as necessidades sentidas pelos cuidadores informais da pessoa com demência é essencial para que se possa estabelecer uma intervenção centrada na pessoa.

No período pré-institucionalização a intervenção dos profissionais de saúde não pode ser descurada, pois é nesta fase que se inicia a relação com a instituição. É fundamental identificar as necessidades de apoio no processo de tomada de decisão e de conhecer e criar confiança nos profissionais da instituiçã̃o. É reconhecida a importância dos cuidadores se sentirem satisfeitos com a instituição escolhida, de forma a gerir os sentimentos de culpa associados à decisão de institucionalização. Afram et al. (2015), Cronfalk et al. (2017) e Graneheim et al. (2014) identificaram situações onde os cuidadores informais não se encontravam preparados para a tomada de decisão de institucionalizar o seu familiar. Ao não conseguirem manter o papel de cuidadores, estes descrevem sentirem-se sozinhos, isolados, com sentimentos de culpa e vergonha. Afram et al. (2015) e Müller et al. (2017) realçam a necessidade de suporte no processo de separação do familiar, embora Afram et al. (2015) tenham identificado a incapacidade dos cuidadores para especificar como gostariam que 0 suporte fosse fornecido. Segundo Graneheim et al. (2014) os cuidadores informais que não conheceram antecipadamente a instituição, expressaram um sentimento de perda de controlo e aumento de stress.

Müller et al. (2017) destacam as intervenções de âmbito psicossocial como potenciadoras da interação com os profissionais, o que pode ter impacto na redução de manifestações de depressão, sobrecarga e instabilidade emocional pelos cuidadores. Destacam que os cuidados devem ser dirigidos para o fortalecimento da interação entre a família e os profissionais de saúde e para a satisfação com a instituição, pois estes dois fatores desencadeiam stress e instabilidade emocional.

Afram et al. (2015), Cronfalk et al. (2017) e Law et al. (2016) evidenciaram que é no momento da visita à pessoa que os cuidadores informais sentem solidão e desamparo.

Afram et al. (2015), Cronfalk et al. (2017), Graneheim et al. (2014) e Law et al. (2016) identificaram a necessidade de disponibilizar um momento de acolhimento à instituição dirigido para os familiares, com um ambiente agradável e acolhedor. Referem-se ao distanciamento entre os profissionais de saúde e os cuidadores informais como uma barreira ao cuidado, que está na origem de sentimentos de desconforto e afastamento.

Law et al. (2016) acrescentam que os cuidadores informais ambicionam sentir-se integrados como parte da instituição e não apenas visitantes, um aspeto essencial ao cuidado centrado na pessoa. Os autores identificaram a importância de desenvolver relações de confiança, de parceria e de partilha de informação, pois querem ver reconhecido o conhecimento que têm sobre a pessoa e a sua experiência de vida. Também Graneheim et al. (2014) e Johansson et al. (2014) realçam o facto de a escassez de informação aumentar o sentimento de insegurança, pelo que ser reconhecido como parceiro no cuidado à pessoa com demência depois da institucionalização, facilita o desenvolvimento da confiança do cuidador. Escutar os cuidadores informais, estar atento às suas dúvidas, pedidos e preocupações é essencial, pois possibilita aos prestadores de cuidados a gestão de cuidados segundo a individualidade da pessoa (Graneheim et al., 2014).

Afram et al. (2015) e Law et al. (2016) verificaram a valorização das interações positivas pelos cuidadores informais. Alguns relatos expressavam baixas expectativas baseadas em contactos anteriores com profissionais de saúde que influenciaram indiretamente a nova experiência, pela diminuição do nível de confiança.

Foi identificada por Afram et al. (2015), Graneheim et al. (2014) e Law et al. (2016) a necessidade dos cuidadores analisarem a formação adequada e a competência dos profissionais para cuidar da pessoa com demência. Quando não há clareza sobre esta competência, os familiares assumem uma postura de monitorização sobre 0 cuidado prestado ao seu familiar. Estes resultados sao partilhados por Graneheim et al. (2014) e Johansson et al. (2014), que verificaram a necessidade do cuidador informal ser supervisor do cuidado prestado, através da 


\section{NECESSIDADES DOS CUIDADORES INFORMAIS DA PESSOA COM DEMÊNCIA EM CONTEXTO DE INSTITUCIONALIZAÇÃO DE LONGO PRAZO: REVISÃO SCOPING}

observação da forma como os clientes foram inseridos na dinâmica da instituição, do cuidado direto prestado, do nível de formação dos prestadores e do respeito pela individualidade da pessoa.

Afram et al. (2015), Cronfalk et al. (2017) e Law et al. (2016) constataram que um baixo rácio cliente/profissionais de saúde e a rotatividade dos prestadores de cuidados constituem um obstáculo à qualidade dos cuidados.

Por outro lado, Graneheim et al. (2014) referem que para os cuidadores informais a institucionalização permitiu reduzir os recursos financeiros e facilitou a gestão da vida familiar, profissional e manter a visita de forma regular ao familiar institucionalizado.

Após análise dos artigos selecionados identificaram-se diferentes necessidades dos cuidadores informais da pessoa com demência em contexto de institucionalização de longo prazo. Salienta-se a identificação de duas fases distintas (Figura 2), nas quais as necessidades identificadas diferem. A pré-institucionalização relacionada com o processo de tomada de decisão de institucionalizar a pessoa, onde se incluem as necessidades de conhecer previamente a instituição e a existência de uma rede de apoio que suporte a decisão. A pós-institucionalização, diz respeito a todas as necessidades que surgem desde 0 momento de admissão da pessoa na instituição de cuidados a longo prazo, nomeadamente 0 acolhimento, 0 apoio durante as visitas, 0 estabelecimento de uma parceria com os cuidadores formais, a monitorização da qualidade dos cuidados e as questões financeiras.

Figura 2 - Necessidades dos cuidadores informais da pessoa com demência

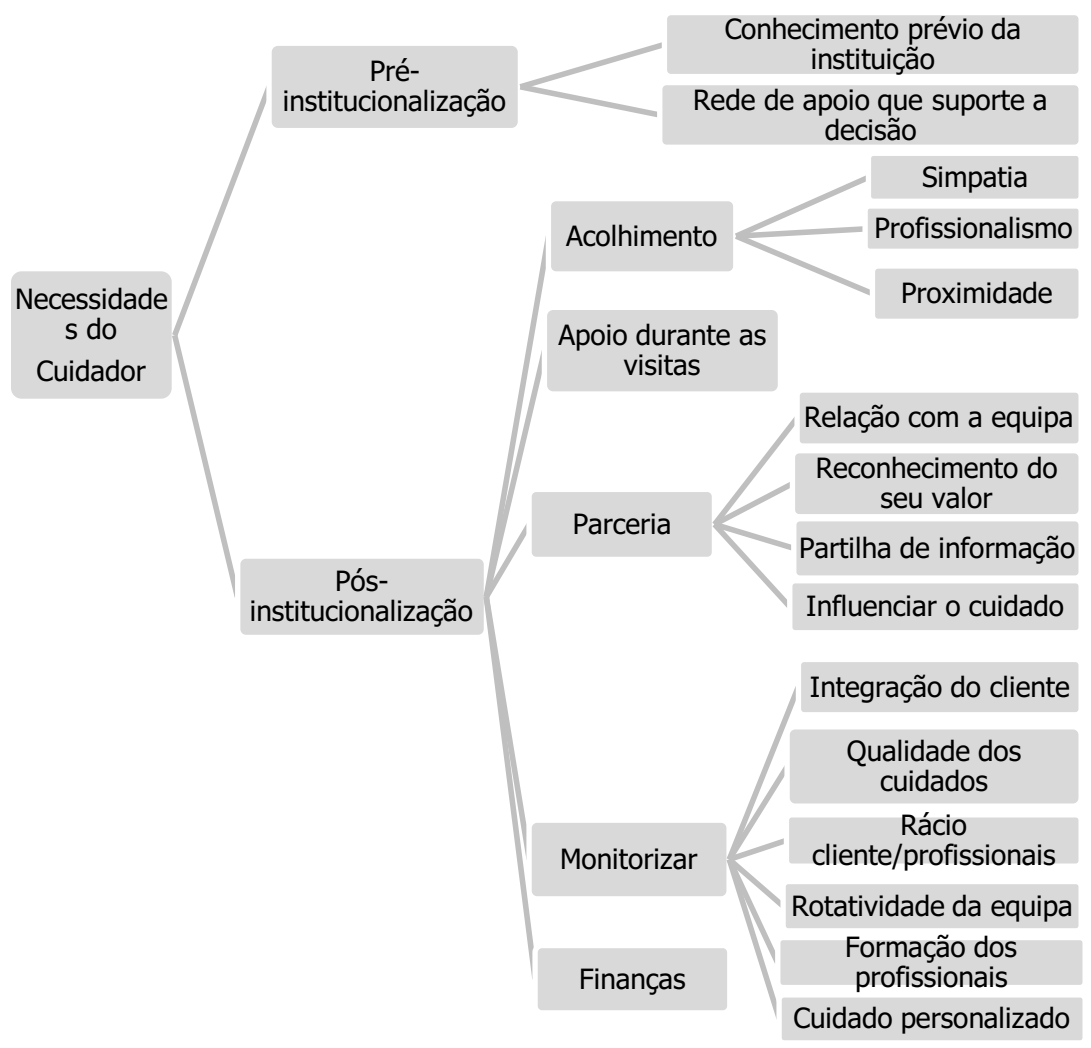


PSICOLOGÍA Y CRECIMIENTO HUMANO

\section{CONCLUSÃO}

A decisão de institucionalizar a pessoa com demência está na origem de focos de necessidades nos cuidadores informais, que requerem intervenção por parte dos profissionais de saúde, de modo a facilitar o seu processo de transição. Necessidades como advogar a vontade da pessoa e monitorizar a qualidade dos cuidados podem ser minimizadas, pois a evidência revela que o seu nível depende da confiança nos profissionais de saúde.

Tendo por fundamento a prática de um cuidado centrado na pessoa, deve ser realizada uma abordagem multidimensional e holística, incluindo os cuidadores informais como alvo da prática do cuidar. A pessoa pelo ser único que é, deve ver respeitada a sua individualidade e ser contemplada numa perspetiva integradora de cuidados, que pode ser alcançada através da definição de um projeto de saúde que englobe uma intervenção de âmbito familiar.

Constatou-se que nenhum dos estudos incluidos foi desenvolvido em Portugal e tendo em conta a pertinência da realização de estudos de investigação sobre o fenómeno em causa, esta revisão contribui para o desenvolvimento do conhecimento sobre o tema.

Como principais fragilidades destaca-se a limitação das bases de dados selecionadas e a janela temporal dos últimos 10 anos definida pelos autores, no sentido procurar um olhar atualizado sobre o fenómeno atraves de artigos científicos recentes. De referir também a limitação da análise de textos redigidos em inglês, espanhol e português, e o facto de não terem sido obtidos resultados com critérios de inclusão da pesquisa de literatura cinzenta, como aspetos de grande influência nos resultados obtidos.

\section{REFERÊNCIAS BIBLIOGRÁFICAS}

Afram, B., Verbeek, H., Bleijlevens, M. H., \& Hamers, J. P. (2015). Needs of informal caregivers during transition from home towards institutional care in dementia: a systematic review of qualitative studies, Int. Psychogeriatric, 27(6), 891-902. D0I: 10.1017/S1041610214002154.

Cronfalk, B., Ternestedt, B., \& Norberg, A. (2017). Being a close family member of a person with dementia living in a nursing home, Journal of Clinical Nursing, 26(21-22), 1-10. D0I: 10.1111/jocn.13718.

Gaugler, J. E., Mittelman, M.S., Hepburn, K., \& Newcomer, R. (2009). Predictors of change in caregiver burden and depressive symptoms following nursing home admission, Psychol Aging, 24(2), 385-396. D0I: $10.1037 / \mathrm{a} 0016052$.

Graneheim, U., Johansson, A., \& Lindgren, B. (2014). Family caregivers' experiences of relinquishing the care of a person with dementia to a nursing home: insights from a meta-ethnographic study, Scandinavian Journal of Caring Sciences, 28, 215-224. DOI: 10.1111/scs.12046.

Hainstock, T., Cloutier, D., \& Penning, M. (2017). From home to 'home': Mapping the caregiver journey in the transition from home care into residential care, Journal of Aging Studies, 43, 32-39. D0I: 10.1016/j.jaging.2017.09.003.

Hoffmann F., \& Rodrigues, R. (2010). Informal carers: who takes care of them? European Centre for Social Welfare Policy and Research, Viena. Disponível em: http://www.euro.centre .org/detail.php?xml_id=1714

Joanna Briggs Institute (2015). Joanna Briggs Institute Reviewers'Manual: 2015 edition / Supplement. The Joana Briggs Institute. The Joanna Briggs Institute. Disponivel em: https://nursing.Isuhsc.edu/JBI/docs/Reviewers Manuals/Scoping-.pdf

Johansson, A., Ruzin, H. 0., Graneheim, U. H., \& Lindgren, B. (2014). Remaining connected despite separation - former family caregivers' experiences of aspects that facilitate and hinder the process of relinquishing the care of a person with dementia to a nursing home, Aging \& Mental Health, 18(8), 1029-1036. D0I: 10.1080/13607863.2014.908456.

Joling, K. J., van Marwijk, H. W., Veldhuijzen, A. E., van der Horst, H. E., Scheltens, P., Smit, F., \& van Hout, H. P. (2015). The two-year incidence of depression and anxiety disorders in spousal caregivers of persons with dementia: who is at the greatest risk? Am J Geriatr Psychiatry, 23(3), 293-303. D0I: 10.1016/j.jagp.2014.0 5.005 . 
Law, K., Patterson, T., \& Muers, J. (2016). Staff Factors Contributing to Family Satisfaction with Long-Term Dementia Care: A Systematic Review of the Literature, Clinical Gerontologist, 40(5), 326-351. D0I: 10.1080/07317115.2016.1260082.

Meleis, A. (2010). Transitions Theory: Middle Range and Situation Specific Theories in Nursing Research and Practice. New York: Springer Publishing Company.

Muller, C., Lautenschläger, S., Meyer, G., \& Stephan, A. (2017). Interventions to support people with dementia and their caregivers during the transition from home care to nursing home care: A systematic review, International Journal of Nursing Studies, 71, 139-152. D0I: 10.1016/j.ijnurstu.2017.03.013.

Organização para Cooperação e Desenvolvimento Económico. (2017). Health at a Glance 2017. Paris: OECD Publishing. Disponível em: https://ec.europa.eu/health/sites/health/ files/state/docs/2018_healthatglance_rep_en.pdf

Pereira, H. J. A. R., (2011). Subitamente cuidadores informais! A experiência de transição para o papel de cuidador informal a partir de um evento inesperado. (Tese de doutoramento). Universidade de Lisboa, Portugal. Disponível no RCAAP http://repositorio.ul.pt/bitstream/10451/3916/1/ulsd61020_td_Helder_Pereira.pdf

Pinquart, M., \& Sörensen, S. (2003). Differences between caregivers and noncaregivers in psychological health and physical health: a meta-analysis, Psychol Aging, 18(2), 250-267. DOI: 10.1037/0882-7974.18.2.250.

Prince, M., Wimo, A., Guerchet, M., Ali, G. C., Wu, Y.T., \& Prina, M. (2015). The Global Impact of Dementia: An Analysis of Prevalence, Incidence, Cost and Trends - World Alzheimer Report 2015. Londres: Alzheimer's Disease International. Disponível em: https://www.alz.co.uk/research/WorldAlzheimerReport2015.pdf

Santana, I., Farinha, F., Freitas, S., Rodrigues, V., \& Carvalho, A. (2015). Epidemiologia da Demência e da Doença de Alzheimer em Portugal: Estimativas da Prevalência e dos Encargos Financeiros com a Medicação, Acta Médica Portuguesa, 28(2), 182-188.

Sury, L., Burns, K., \& Brodaty, H. (2013). Moving in: adjustment of people living with dementia going into a nursing home and their families, International Psychogeriatrics, 25, 867-876. D0I: 10.1017/S10416102130000 57.

Thompson, G. N., \& Roger, K. (2014). Understanding the needs of family caregivers of older adults dying with dementia, Palliative \& Supportive Care, 12, 223-231. D0I: 10.1017/S1478951513000461. 